\title{
The Value of Chinese Version GAD-7 and PHQ-9 to Screen Anxiety and Depression in Chinese Outpatients with Atypical Chest Pain
}

\author{
Qiuzhen Lin ${ }^{1-4}$ \\ Ousseina Bonkano ${ }^{1-5}$ \\ Keke $\mathrm{Wu}^{\mathrm{I}-4}$ \\ Qiming Liu ${ }^{1-4}$ \\ Toure Ali Ibrahim ${ }^{5}$ \\ Ling Liu ${ }^{1-4}$
}

'Department of Cardiovascular Medicine, The Second Xiangya Hospital, Central South University, Changsha, People's Republic of China; ${ }^{2}$ Research Institute of Blood Lipid and Atherosclerosis, Central South University, Changsha, People's Republic of China; ${ }^{3}$ Modern Cardiovascular Disease Clinical Technology Research Center of Hunan Province, Changsha, People's Republic of China; ${ }^{4}$ Cardiovascular Disease Research Center of Hunan Province, Changsha, Hunan, 4I00II, People's Republic of China; ${ }^{5}$ Department of Cardiovascular and Internal Medicine Niger, Niamey Amirou Boubacar Diallo National Hospital, Abdou Moumouni University, Niamey, Niger
Correspondence: Ling Liu

Department of Cardiovascular Medicine,

The Second Xiangya Hospital, Central

South University, \#I39 Middle Renmin

Road, Changsha, Hunan, 4I00II, People's

Republic of China

Fax +86-73।85295407

Email feliuling@csu.edu.cn
Background: Atypical chest pain in some outpatients could derive from mental disorders. It is necessary for them to have a preliminary emotional assessment in the outpatient department of Cardiology before psychiatric outpatient visits.

Methods: This study included 122 Chinese outpatients with atypical chest pain in the department of Cardiology. They accepted routine examinations, including treadmill test, and were judged by the three-question method as highly likely to have emotional disorders. Then, a standard questionnaire package containing the Chinese version of the seven-item scale for General Anxiety Disorder (GAD-7), Self-rating Anxiety Scale (SAS), the nine-item Patient Health Questionnaire (PHQ-9) and Self-rating Depression Scale (SDS) was administered to evaluate anxiety and depression.

Results: The percentages of anxiety evaluated by GAD-7 and SAS were $62.3 \%$ and $26.2 \%$, respectively. Analogously, the assessment by PHQ-9 showed a significantly higher percentage of depression than that by SDS $(61.5 \%$ vs $29.5 \%)(P<0.05)$. Kappa analysis showed that the consistency between GAD-7 and SAS, or that between PHQ-9 and SDS was not very good. About $73 \%$ outpatients suffered from emotional disorders, presenting as anxiety/ depression evaluated by GAD-7 and PHQ-9. Furthermore, sleep disorders accounted for more than $80 \%$ of patients with mental disorders. Finally, the suicidal tendency of depression patients was about $17 \%$ that should not be ignored.

Conclusion: Compared with SAS and SDS, GAD-7 and PHQ-9 detected more participants with emotional disorders in the Chinese outpatients with atypical chest pain, indicating that GAD-7 and PHQ-9 could be briefly well-validated tools to screen emotional disorders in the outpatient department of Cardiology before psychiatric visits.

Keywords: depression, anxiety, screen, atypical chest pain

\section{Introduction}

Chest pain is the discomfort or pain that someone feels anywhere along the front of body between neck and upper abdomen. Any organ or tissue, such as esophagus, heart and nerve, in the chest can be the source of pain. However, not all chest pain has an organic etiology. The new diagnosis of chronic primary pain in the International Classification of Diseases 11 (ICD-11) is when pain has persisted for more than 3 months and is associated with significant emotional distress and/or functional disability, and the pain is not better accounted for by another condition. ${ }^{1}$ Atypical chest pain is recurrent angina pectoris-like pain without evidence of coronary heart disease in conventional diagnostic evaluation. ${ }^{2,3}$ Many studies have shown that atypical chest 
pain cannot be determined by an organic etiology. It could be a component or an accompanying symptom of a specific psychiatric disorder most of the time. ${ }^{4-7}$ At the end of last century, substantial studies had demonstrated that recurrent chest pain in some patients were caused by mental illness in Western countries. ${ }^{6,8-12}$ The prevalence of anxiety among patients with non-cardiac chest pain in Western countries reached more than $30 \% .^{13,14}$ Similar studies were rare in China at that time.

Recently, a dramatic increase in the prevalence of mental disorders was revealed in China. ${ }^{15,16}$ One study conducted in Hong Kong indicated that 5.0\%, 15.8\%, and $10.0 \%$ of the general population were identified as having somatic, psychological, and mixed distress, respectively. And the proportions of low distress reached to $69.2 \% .{ }^{17}$ On the other hand, Chinese patients with psychological distress alone sought less help from psychological doctors, despite their severe psychological impairment. ${ }^{18}$ Furthermore, Chinese people, including Chinese Americans and Chinese Malaysian (Chinese immigrants or their descendants), were more likely to express somatic symptoms rather than emotional distress. ${ }^{19,20}$ Thus, such patients gathered in the comprehensive departments but not spiritual psychological department, and their diagnosis became challenging.

A series of self-rating scales have been used to determine the emotional state of those suspected patients with unexplained discomfort in the comprehensive departments. The scales of Self-rating Anxiety Scale (SAS), Self-rating Depression Scale (SDS), seven-item scale for General Anxiety Disorder (GAD-7) and the nine-item Patient Health Questionnaire (PHQ-9) have been recommended to assess anxiety or depression in primary care setting. ${ }^{21-25}$ In this study, the outpatients with atypical chest pain were screened by three-question method and showed high possibility of emotional disorders, then they were further evaluated by SDS, SAS, GAD-7 and PHQ-9. We aimed to compare the validity between GAD-7/PHQ-9 and SAS/SDS in screening anxiety and/or depression, and to provide better diagnosis for those patients in the outpatient department of cardiovascular clinic.

\section{Methods}

\section{Subjects}

One hundred and twenty two outpatients with atypical chest pain at department of cardiovascular medicine, the Second Xiangya Hospital, Central South University from January 1 to July 30 in 2018 were recruited in this study. The enrolled subjects were the patients who visited our hospital for the first time and they did not take the drugs that could affect their mental status. All subjects were 18 79 years old, including $79(64.8 \%)$ women and 43 (35.2\%) men. Atypical chest pain was defined as non-cardiac or unexplained chest pain. ${ }^{2,3}$ Atypical chest pain occurred in a resting state and was characterized by tingling or dull pain without radiation. It often lasted less than one minute, sometimes more than one hour. Moreover, all subjects accepted treadmill test and the tests of D-Dimer, high sensitivity troponin, creatine kinase, which results were negative, indicating that chest pain was not angina pectoris due to myocardial ischemia. Of course, all participants also accepted routine enquiry, body examination, the examinations of electrocardiogram, chest $\mathrm{X}$ ray and pulmonary function test to exclude organic diseases. According to Chinese expert consensus on psychological prescription for the patients in the cardiovascular department in 2014, it was important to screen psychological problems in patients who were no corresponding organic changes. Thus, 'three questions' needed be provided to those subjects with unexplained chest pain. In this study, patients had chest pain that physicians just asked two questions. ${ }^{26}$ Firstly, do you have poor sleep? Secondly, do you feel anxious? If the answer to at least one of the two questions was 'yes', it suggested that the patient was probably to have mental and psychological problems. Then, anxiety self-rating scales (SAS and GAD-7) and depression self-rating scales (SDS and PHQ-9) were performed to assess their mental status.

Subjects were excluded if they had a previous history of cardiovascular diseases, cerebrovascular diseases, pulmonary diseases, digestive tract diseases, cognitive impairment, psychosis or acute suicidal tendencies.

\section{Assessment Instruments GAD-7}

It was a seven-item, self-rated scale developed by Spitzer and colleagues ${ }^{27}$ as a screening tool and severity indicator for generalized anxiety disorders in the last two weeks. The instrument showed good reliability and validity in a Chinese population. ${ }^{28,29}$ The GAD-7 score was calculated by assigning scores of $0,1,2$, and 3, corresponding to "not at all", "several days", "more than half the days", and "nearly every day", respectively, and adding together the scores for the seven questions. Scores of 5, 10, and 15 were taken as the cut-off points for "mild", "moderate" and "severe" anxiety, respectively. 


\section{SAS}

It was also a screening instrument for anxiety, which consisted of twenty items that reflected the symptom criteria for general anxiety disorder. ${ }^{30}$ Patients made a self-evaluation for each item on a 4-point scale in order to know how they had been bothered by anxiety symptoms in the last two weeks. Adding together the scores for all the questions. Scores of 53 62, 63 72, and over 72 were used to indicate a "mild", "moderate" and "severe" anxiety, respectively.

\section{PHQ-9}

It was used to evaluate the severity of depression in the last two weeks through nine questions. Each item was rated on a 4 grades ranging from 0 (not at all) to 3 (nearly every day). A total score (0-27) was obtained by summing across all items. Higher scores indicated higher levels of anxiety symptoms. Cut-off values of 5, 10 and 15 represented "mild", "moderate" and "severe" depression, respectively. 31,32

\section{SDS}

It was also employed to evaluate depression in the last two weeks. ${ }^{33}$ The brief scale was recommended to provide evidences indicating depression, with higher scores reflecting higher probability of suffering from depression. Cut-off values of 50 59, 60 69, over 70 were used to indicate a "mild", "moderate" and "severe" depression, respectively.

\section{Statistical Analysis}

Statistical analysis was performed on Statistical Package for Social Sciences version 20.0. Data drawing was completed by Origin Pro 8.0 software. Quantitative variables were expressed as mean \pm standard deviation $(M \pm S D)$, and qualitative variables were expressed as numbers and percentages. Differences between the inter-group means were analyzed by $t$-test. Categorical variables were compared using chi-squared statistic tests. Reliability analysis was performed with the Kappa statistic to determine consistency among estimators. Two estimators were considered to be in poor agreement if the Kappa coefficient was $<0.4$, i.e. in slight agreement if 0.00 to 0.20 , fair agreement if 0.21 to 0.40 , moderate agreement if 0.41 to 0.60 , substantial agreement if 0.61 to 0.80 , and in perfect agreement if it was 0.81 to $1.00 .^{34}$ All $P$ values were 2-tailed, and $P<0.05$ was considered statistically significant.

\section{Results}

\section{Emotional Disorders Evaluated by Different Self-Rating Scales}

The percentages of anxiety evaluated by GAD-7 and SAS were $62.3 \%$ and $26.2 \%$, respectively. The number of patients diagnosed as anxiety by GAD-7 was significantly higher than that by SAS $(P<0.05)$. The percentage of moderate $(21.3 \%$ vs $4.9 \%$ ) or severe ( $15.6 \%$ vs $2.5 \%$ ) anxiety by GAD-7 was significantly higher than that by SAS $(P<0.0167)$.

Analogously, the assessment by PHQ-9 showed a significantly higher percentage of depression when compared to that by SDS $(61.5 \%$ vs $29.5 \%)(P<0.05)$. The percentages of patients diagnosed with low $(35.2 \%$ vs $21.3 \%$ ), moderate (16.4\% vs $5.7 \%$ ) and severe $(9.8 \%$ vs $2.5 \%$ ) depression by PHQ-9 were also in higher levels than those by $\operatorname{SDS}(P<0.0167)$ (Table 1$)$.

Kappa analysis was performed to determine the consistency of GAD-7 and SAS in the diagnosis of anxiety, and that of PHQ9 and SDS in the diagnosis of depression. There was in fair agreement between SAS and GAD-7 in the diagnosis of anxiety because Kappa coefficient was only 0.295 . Moreover, the value for Kappa was 0.356 indicating a fair level of agreement between SDS and PHQ-9 in the diagnosis of depression. In other words, the consistency between GAD-7 and SAS, or that between PHQ9 and SDS was not very good (Data were not shown).

\section{Sociodemographic Characteristics}

Considering that GAD-7 and PHQ-9 have recently been recommended for emotional assessment of cardiovascular outpatients, ${ }^{26}$ the clinical characteristics of patients with emotional disorders diagnosed by these two self-rating scales were analyzed. All subjects were classified to patients with anxiety and/or depression and those without either disorder. There was no significant difference in age, sex, blood pressure, heart rate, residence, marital status, life situations, family income, occupation and education between two groups (Table 2).

\section{Clinical Manifestations}

The most common clinical manifestation was sleep disorder, accounting for $83 \%$ of patients with emotional disorders. Followed by chest distress, more than $50 \%$. Palpitation and shortness of breath accounted for nearly $30 \%$.

In outpatients without anxiety or depression, the percentages of sleep disorder and chest distress were also relatively high. Although these patients were not diagnosed with emotional disorders by GAD-7 and PHQ-9, it suggested the possibility of somatic disorder (Table 3 ). 
Table I Anxiety and Depression Evaluated by Different Self-Rating Scales

\begin{tabular}{|c|c|c|c|c|c|c|c|c|}
\hline & \multicolumn{2}{|c|}{ GAD-7 } & \multicolumn{2}{|c|}{ SAS } & \multicolumn{2}{|c|}{ PHQ-9 } & \multicolumn{2}{|c|}{ SDS } \\
\hline & $\mathbf{n}$ & $\%$ & $\mathbf{n}$ & $\%$ & $\mathbf{n}$ & $\%$ & $\mathbf{n}$ & $\%$ \\
\hline Without anxiety ${ }^{a}$ & 46 & $37.70 \%$ & 90 & $73.80 \%$ & & & & \\
\hline Anxiety total ${ }^{\mathrm{c}}$ & 76 & $62.30 \%$ & 32 & $26.20 \%$ & & & & \\
\hline Low level & 31 & $25.40 \%$ & 23 & $18.90 \%$ & & & & \\
\hline Moderate level & 26 & $21.30 \%$ & 6 & $4.90 \%$ & & & & \\
\hline High level & 19 & $15.60 \%$ & 3 & $2.50 \%$ & & & & \\
\hline Without depression ${ }^{b}$ & & & & & 47 & $38.50 \%$ & 86 & $70.50 \%$ \\
\hline Depression total ${ }^{d}$ & & & & & 75 & $61.50 \%$ & 36 & $29.50 \%$ \\
\hline Low level & & & & & 43 & $35.20 \%$ & 26 & $21.30 \%$ \\
\hline Moderate level & & & & & 20 & $16.40 \%$ & 7 & $5.70 \%$ \\
\hline High level & & & & & 12 & $9.80 \%$ & 3 & $2.50 \%$ \\
\hline
\end{tabular}

Notes: ${ }^{a} P<0.05$ patients with anxiety assessed by GAD-7 when compared to those by SAS. ${ }^{b} P<0.05$ patients with depression assessed by PHQ-9 when compared to those by SDS. ${ }^{c} P<0.05$ different anxiety levels assessed by GAD-7 when compared to those by SAS. ${ }^{d} P<0.05$ different depression levels assessed by PHQ-9 when compared to those by SDS.

Table 2 Sociodemographic Characteristics of Patients with Mental Diseases Assessed by GAD-7 and PHQ-9

\begin{tabular}{|c|c|c|c|}
\hline & Anxiety and/or Depression $(n=89)$ & Both Negative $(n=33)$ & $\mathbf{P}$ \\
\hline Age $(M \pm S D)$ & $50.70 \pm 10.21$ & $52.67 \pm 11.69$ & 0.397 \\
\hline Female (n, \%) & $57(64)$ & $22(66.7)$ & 0.843 \\
\hline $\mathrm{SBP}(\mathrm{mmHg})(\mathrm{M} \pm \mathrm{SD})$ & $130.73 \pm 12.43$ & $131.36 \pm 17.59$ & 0.825 \\
\hline $\mathrm{DBP}(\mathrm{mmHg})(\mathrm{M} \pm \mathrm{SD})$ & $75.13 \pm 10.84$ & $75.03 \pm 10.45$ & 0.961 \\
\hline HR (beats/min) $(M \pm S D)$ & $78.65 \pm 13.85$ & $80.84 \pm 10.39$ & 0.416 \\
\hline Residence (n, \%) & & & 0.066 \\
\hline City & $34(38.2)$ & $19(57.6)$ & \\
\hline Rural & $55(61.8)$ & $14(42.4)$ & \\
\hline Marital status (n, \%) & & & 0.514 \\
\hline Single & $8(9.1)$ & I (3.I) & \\
\hline Married & $77(86.5)$ & $30(90.9)$ & \\
\hline Divorced/Widowed & $4(4.4)$ & $2(6.1)$ & \\
\hline Life situation $(n, \%)$ & & & 0.757 \\
\hline Alone & $12(13.6)$ & $3(9.4)$ & \\
\hline With others & $77(86.4)$ & $30(90.6)$ & \\
\hline Monthly family income (n, \%) & & & 0.134 \\
\hline Less than 4000RMB & $65(72.7)$ & $21(62.5)$ & \\
\hline 4000-8000RMB & $16(18.2)$ & II (34.4) & \\
\hline More than 8000RMB & $8(9.1)$ & I (3.I) & \\
\hline Occupation (n, \%) & & & 0.686 \\
\hline Employed/student & $50(56.2)$ & $20(60.6)$ & \\
\hline Unemployed & $39(43.8)$ & $13(39.4)$ & \\
\hline Education (n, \%) & & & 0.182 \\
\hline Elementary & $42(47.3)$ & $18(54.5)$ & \\
\hline Middle/high school & $46(51.6)$ & $13(39.4)$ & \\
\hline University or higher & I (I.I) & $2(6.1)$ & \\
\hline
\end{tabular}

Abbreviations: RMB, the renminbi is the currency of the People's Republic of China; SBP, systolic blood pressure; DBP, diastolic blood pressure; HR, heart rate. 
Table 3 Clinical Manifestations of Patients

\begin{tabular}{|l|c|c|c|c|c|}
\hline & \multicolumn{2}{|c|}{ Anxiety and/or Depression (n=89) } & \multicolumn{2}{|c|}{ Both Negative (n=33) } & $P$ \\
\hline Chest distress (n, \%) & 48 & 53.9 & 15 & 46.9 & 12.5 \\
Palpitation (n, \%) & 24 & 27 & 4 & 15.6 & 0.54 \\
Dyspnea (n, \%) & 24 & 27 & 5 & 25 & 0.142 \\
Headache/Dizziness (n, \%) & 13 & 14.6 & 8 & 62.5 & 0.186 \\
Sleep disorder (n, \%) & 73 & 83 & 20 & 0.03 \\
\hline
\end{tabular}

Furthermore, those subjects without anxiety or depression by GAD-7 and PHQ-9 were then evaluated by SAS and SDS, there was also no evidence of emotional disorders (Data was not shown).

\section{The Positive Rate of Each Item in GAD-7 and PHQ-9}

For that the subjects in this study were the outpatients suspected to have emotional disorders in the Department of cardiovascular medicine, the positive rate of each item in GAD-7 and PHQ-9 was analyzed when taking all subjects as a whole. The positive rate of each item in GAD-7 was more than $40 \%$. The positive rate of item 6 , the question about
"Becoming easily annoyed or irritable?" was the highest, which was more than $70 \%$. The second highest was item 1 , the question about "Feeling nervous, anxious or on edge?", which was near to $70 \%$. Then followed by item 3 , the question about "Worrying too much about different things?", which was more than 60\% (Figure 1A).

The positive rate of each item in PHQ-9 differed greatly. The positive rates of item 1-4 in PHQ-9 exceeded $40 \%$. The positive rate of item 3 , the question about "Trouble falling or staying asleep, or sleeping too much?" was the highest, which was more than $70 \%$. The second highest was item 4, the question about "Feeling tired or having little energy?", which was near
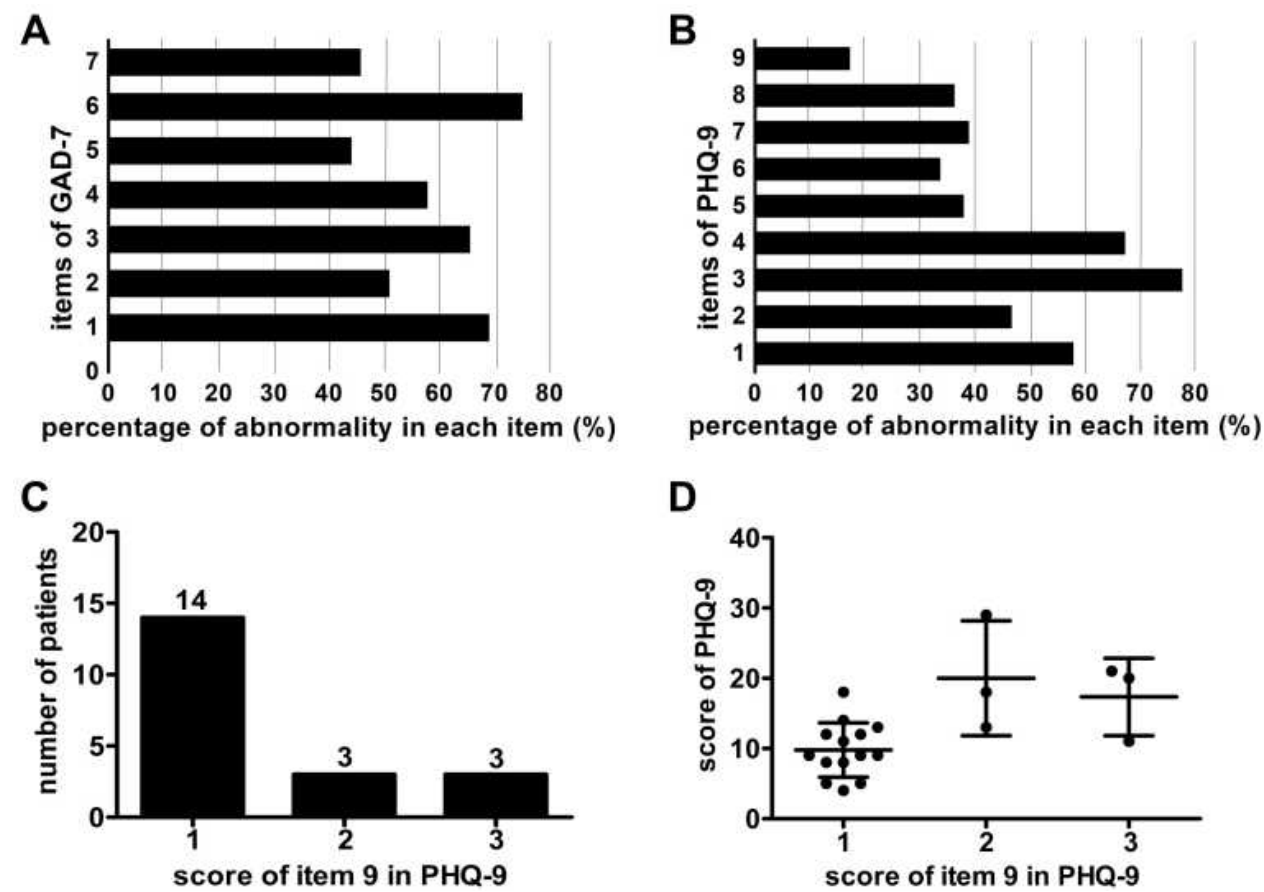

D

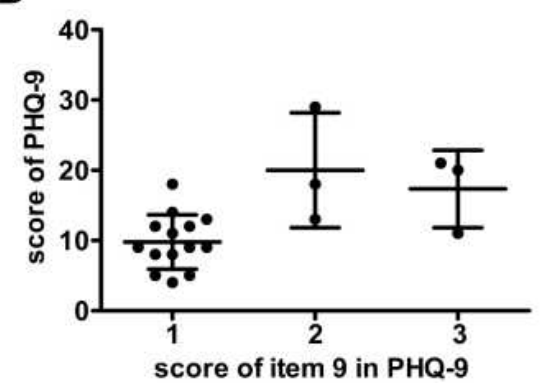

Figure I Abnormality in each item of GAD-7 and PHQ-9. (A) The percentage of abnormality of each item in GAD-7. The items were as follows: I. Feeling nervous, anxious or on edge? 2. Not being able to stop or control worrying? 3. Worrying too much about different things? 4. Trouble relaxing? 5 . Being so restless that it is hard to sit still? 6. Becoming easily annoyed or irritable? 7. Feeling afraid as if something awful might happen? (B) The percentage of abnormality in each item of PHQ-9. The items were as follows: I. Little interest or pleasure in doing things? 2. Feeling down, depressed, or hopeless? 3. Trouble falling or staying asleep, or sleeping too much? 4. Feeling tired or having little energy? 5. Poor appetite or overeating? 6. Feeling bad about yourself or that you are a failure or have let yourself or your family down? 7. Trouble concentrating on things, such as reading the newspaper or watching television? 8. Moving or speaking so slowly that other people could have noticed? Or the opposite being so fidgety or restless that you have been moving around a lot more than usual? 9. Thoughts that you would be better off dead, or of hurting yourself in some way? (C) Number of patients with item 9 score which was greater than 0. (D) Total scores of PHQ-9 in different item 9 scores. 
to $70 \%$. Then followed by item 1 , the question about "Little interest or pleasure in doing things?", which was more than $50 \%$ (Figure 1B).

The positive rate of item 9, the question about "Thoughts that you would be better off dead, or of hurting yourself in some way", was $17.2 \%(n=20)$ (Figure 1B). Considering the particularity of item 9 of PHQ-9, we focused on if there was any patient with PHQ-9 total score $<5$ but the score of item $9 \geq 1$, and finally found out one such a patient. However, this patient was also judged to be emotional disorder because of anxiety evaluated by GAD-7. Of 14 patients with item 9 score $=1$, only one patient had a total score of PHQ-9 $\geq 15$. Of 6 patients with Item 9 score $=2$ or 3, 4 patients had PHQ-9 score $\geq 15$ (Figure 1C and D).

\section{Discussion}

To evaluate the emotional disorders in outpatients with atypical chest pain in the department of cardiovascular medicine, two different self-rating scales were used to screen anxiety and depression in this study. GAD-7 or PHQ-9 showed a significantly higher percentage of anxiety or depression when compared with SAS or SDS, respectively. More importantly, kappa analysis suggested that the agreement between those two sets of self-rating scales was not very good. This could be the first study to compare GAD-7/PHQ-9 and SAS/SDS in screening emotional disorders among Chinese cardiovascular outpatients. The corresponding research results could have a certain guiding value for the clinical screening of emotional disorders in non-psychiatric outpatient clinic from general hospitals.

In recent years, the departments of cardiovascular medicine, neurology and gastroenterology in general hospitals have become the popular departments for Chinese patients with emotional disorders to seek help outside the psychological center. ${ }^{35-39}$ With the deepening understanding of emotional disorders, more and more scholars reported the emotional problems of cardiovascular outpatients or inpatients. ${ }^{35-37}$ There are two types of patients with emotional disorders in the department of cardiovascular medicine. Some patients have been diagnosed with cardiovascular diseases and complicated with emotional disorders. Others are just patients with suspected cardiovascular symptoms. The patients enrolled in this study belonged to the latter type. The visits of these patients present great challenges to cardiovascular physicians. They should not only master and apply cardiovascular professional knowledge to help the patients, but also understand the clinical manifestations and screening of emotional disorders.

Self-rating scale is a powerful assistant for cardiovascular physicians to identify the suspected patients with emotional disorders, because the non-psychiatric doctors are not qualified to use non-self-rating scale. There are many kinds of self-rating scales, including GAD-7/PHQ-9, SAS/SDS, and so on. In addition, there are simpler ones with two or three questions. For example, patient health questionnaire-2 (PHQ-2) contains only two questions that can be used for rapid screening of patients with suspected depression. ${ }^{40}$ In this study, we used three-question method that is recommended by the consensus of Chinese cardiovascular experts to screen the patients with atypical chest pain. ${ }^{26}$ If two or more answers to three questions were yes, the patient should be recommended for further selfassessment, such as evaluating by GAD-7/PHQ-9 or SAS/SDS. Among the three questions, the third one that should be paid attention to is whether the patient has obvious but unexplained physical discomfort. In this process, physicians are required to determine whether the patient with chest pain has an organic etiology or not. For the patients with atypical chest pain in this study, they underwent exercise tests but did not get positive results of myocardial ischemia. Combining with the presence of sleep disorders and/or anxious condition, there was the possibility of emotional disorders in these patients.

Interestingly, the proportion of patients with emotional disorders found by the two self-rating scales was quite different. SAS or SDS reported less than 30\% patients with anxiety or depression, respectively, while GAD-7 or PHQ-9 discovered more than $60 \%$ patients with anxiety or depression, respectively, if only according to the total scores. This difference may be caused by several factors. First, SAS or SDS may have a relatively low positive detection rate. Previous studies also showed a lower ratio, $<30 \%$, of emotional disorders in patients with cardiovascular diseases evaluated by SAS or SDS. ${ }^{35,37}$ Second, for the suspected patients with emotional disorders, the number of items in the selfrating scales may affect the completion of the scales to a certain extent. The more items there are, the more difficult it is for the patient to focus on the scales, especially in the outpatient department. There are as many as 20 items in SAS or SDS. Due to the relatively fewer items of GAD-7 or PHQ9, patients are more easy to focus on all items and quickly complete self-rating in a short time. According to the score alone, $73 \%$ of the patients had anxiety and/or depression. It 
was close to the proportion of patients with emotional disorders evaluated by GAD-7/PHQ-9 scales in cardiovascular outpatient clinic reported by other Chinese scholars. ${ }^{41}$ These data suggested that GAD-7 or PHQ-9 scale may be an ideal screening tool for suspected emotional disorders in Chinese cardiovascular clinics.

It is undeniable that only relying on the total score of PHQ-9 scale to determine depression may lead to some missed diagnosis. In this study, twenty patients expressed the emotion of wanting to die or self-injuring, but not all total score of them showed the level of severe depression, i.e. $\geq 15$, and even some patients had scores $<5$. For patients with the tendency of suicide or self-mutilation, regardless of the total score, they should be transferred to the psychological center under strict supervision as fast as possible for further evaluation and active intervention, just like the patients who had severe depression according to the total score $\geq 15$.

We noticed that about one-fourth (33/122) of the patients in this study showed no sign of emotional disorder, but they had a high proportion of sleep or somatization disorders. Sleep and mental health are closely connected and influence each other. Sleep disorders may increase risk for developing mental disorders, as well as may only a manifestation of mental diseases. Patients with sleep disorders are more likely to alter their emotional status, and those with mental diseases are more likely to have sleep problems. Furthermore, patients with psychiatric disorders (50\% to $80 \%$ ) are more likely to suffer from sleep disorders than general population (10\% to $18 \%){ }^{42}$ However, the mutual relationship between sleep and mental health is not yet completely understood. Therefore, when sleep disorders and mental disorders are mutually causative and difficult to identify the cause, physician and psychiatrist can work together to distinguish which is the cause.

Compared with extroverted Westerners, Asians are more introverted and are not good at or willing to express their inner feelings. Due to the misunderstanding of emotional disorders, they even feel ashamed or reject to go to the psychiatric center to receive evaluation. Even if they were assessed by the self-rating scales in the cardiovascular clinic, they were likely to hide their true thoughts. On the contrary, they would repeatedly emphasize their physical discomfort. In this case, somatization scales, such as PHQ15 , could help identify such patients. According to Yu et al, the total detection rate of anxiety and/or depression in somatization disorder patients was $78.6 \%$, and $21.4 \%$ of patients were negative. ${ }^{41}$ Therefore, patients with negative results by GAD-7 and PHQ-9 scales should be further evaluated with somatization scale, such as PHQ-15.

\section{Limitations}

This paper had its limitations: firstly, the number of the study sample was small. Secondly, it would be more illustrative of the severity of somatic symptoms if this study were done with PHQ-15. Therefore, PHQ-15 can be used in future studies to evaluate psychiatric situations in patients with atypical chest pain but without anxiety/ depression.

\section{Conclusions}

This study showed a high prevalence of mental disorders in patients with atypical chest pain. Compared with SAS and SDS, GAD-7 and PHQ-9 detected more participants with emotional disorders in the Chinese outpatients with atypical chest pain, indicating that GAD-7 and PHQ-9 could be briefly well-validated tools to screen emotional disorders before psychiatric outpatient visits. In addition, sleep disorder and suicidal thoughts should be paid closely attention in patients with atypical chest pain that cannot be explained by an organic etiology. The corresponding research results could have a certain guiding value for the clinical screening of emotional disorders in nonpsychiatric outpatient clinic from general hospitals.

\section{Abbreviations}

SAS, the scales of Self-rating Anxiety Scale; SDS, Selfrating Depression Scale; GAD-7, seven-item scale for General Anxiety Disorder; PHQ-9, the nine-item Patient Health Questionnaire; PHQ-2, Patient Health Questionnaire-2; SD, standard deviation; SBP, systolic blood pressure; DBP, diastolic blood pressure; HR, heart rate; RMB, the currency of the People's Republic of China.

\section{Ethics Statement}

All procedures performed in this study involving human participants were in accordance with the ethical standards of the institutional and/or national research committee and with the 1964 Helsinki declaration and its later amendments or comparable ethical standards. Anonymised data were used in the evaluation of the GAD-7/PHQ-9 and $\mathrm{SAS} / \mathrm{SDS}$. The data collection procedure was discussed and approved by the Ethics Committee of the Second Xiangya Hospital of Central South University. This was a cross-sectional study that only analyzed patients' data, so we did not received the formal written consent from the ethics committee. 


\section{Credit Authorship Contribution Statement}

Qiuzhen Lin: Created the tables and figures, interpreted the results, and contributed to the preparation of all components of the manuscript. Bonkano Ousseina: Conceived of the idea and research questions, and obtained the data. Keke Wu: Conducted the analyses. Qiming Liu: Conducted the literature review. Ali Ibrahim Toure: Conducted the literature review. Ling Liu: Conducted the literature review, checked and modified the full text. All authors contributed to data analysis, drafting or revising the article, have agreed on the journal to which the article will be submitted, gave final approval of the version to be published, and agree to be accountable for all aspects of the work. Qiuzhen Lin and Bonkano Ousseina contributed equally to the writing of this article.

\section{Acknowledgments}

We confirmed that the patient data accessed complied with relevant data protection and privacy regulations.

\section{Funding}

This project was supported by the National Natural Science Foundation of China [grant numbers: 81270956, 81470577] and the Fundamental Research Funds for the Central Universities of Central South University [grant number 2020zzts289].

\section{Disclosure}

The authors declared no conflict of interests for this work. The abstract of this paper was presented at the Great Wall International Congress of Cardiology 2020, Asian Heart Society Congress 2020 as a poster presentation with interim findings. The poster's abstract was published in "Poster Abstracts" in Cardiovascular Innovations and Applications Journal name "The value of Chinese version GAD-7 and PHQ-9 to screen anxiety and depression in Chinese outpatients with atypical chest pain": https://www.ingentacon nect.com/content/cscript/cvia/2020/00000005/a00101s1/ art00001;jsessionid=1 vzxwh0u5rcmx.x-ic-live-03.

\section{References}

1. Nicholas M, Vlaeyen JWS, Rief W, et al. The IASP classification of chronic pain for ICD-11: chronic primary pain. Pain. 2019;160 (1):28-37. doi:10.1097/j.pain.000000000001390

2. van Peski-oosterbaan AS, Spinhoven $P$, van der Does AJ, Bruschke AV. Noncardiac chest pain: interest in a medical psychological treatment. J Psychosom Res. 1998;45(5):471-476. doi:10.1016/ S0022-3999(97)00306-1
3. Esler JL, Bock BC. Psychological treatments for noncardiac chest pain: recommendations for a new approach. $J$ Psychosom Res. 2004;56(3):263-269. doi:10.1016/S0022-3999(03)00515-4

4. Bass C, Wade C. Chest pain with normal coronary arteries: a comparative study of psychiatric and social morbidity. Psychol Med. 1984;14(1):51-61. doi:10.1017/S003329170000307X

5. Chambers J, Bass C. Chest pain with normal coronary anatomy: a review of natural history and possible etiologic factors. Prog Cardiovasc Dis. 1990;23:161-184. doi:10.1016/0033-0620(90)90007-O

6. Beitman BD, Mukerji V, Lamberti JW, et al. Panic disorder in patients with chest pain and angiographically normal coronary arteries. Am J Cardiol. 1989;63(18):1399-1403. doi:10.1016/0002-9149(89)91056-4

7. Hocaoglu C, Gulec MY, Durmus I. Psychiatric comorbidity in patients with chest pain without a cardiac etiology. Isr J Psychiatry Relat Sci. 2008;45(1):49-54.

8. Katon W, Hall ML, Russo J, et al. Chest pain: relationship of psychiatric illness to coronary arteriographic results. Am J Med. 1988;84(1):1-9. doi:10.1016/0002-9343(88)90001-0

9. Latinga LJ, Sprafkin RP, McCroskey JH, et al. One-year psychosocial follow-up of patients with chest pain and angiographically normal coronary arteries. Am J Cardiol. 1988;62(4):209-213. doi:10.1016/ 0002-9149(88)90213-5

10. Tueth MJ. Managing recurrent nonischemic chest pain in the emergency department. Am J Emerg Med. 1997;15(2):170-172. doi:10.1016/S07356757(97)90094-2

11. Carter CS, Servan-Schreiber D, Perlstein WM. Anxiety disorders and the syndrome of chest pain with normal coronary arteries: prevalence and pathophysiology. J Clin Psychiatry. 1997;58(suppl3):70.

12. Fleet RP, Dupuis G, Marchand A, et al. Panic disorder in coronary artery disease patients with noncardiac chest pain. J Psychosom Res. 1998;44(1):81-90. doi:10.1016/S0022-3999(97)00136-0

13. Carter C, Maddock RJ. Chest pain in generalized anxiety disorder. Int J Psychiatry Med. 1992;22(3):291-298. doi:10.2190/RGC5-PJK2 -7TG5-KL1B

14. Cormier LE, Katon W, Russo J, Hollifield M, Hall ML, Vitaliano PP. Chest pain with negative cardiac diagnostic studies. Relationship to psychiatric illness. J Nerv Ment Dis. 1988;176(6):351-358. doi:10.1097/00005053-198806000-00004

15. Guo WJ, Tsang A, Li T, Lee S. Psychiatric epidemiological surveys in China 1960-2010: how real is the increase of mental disorders? Curr Opin Psychiatry. 2011;24(4):324-330. doi:10.1097/YCO.0b013e3283477b0e

16. Phillips MR, Zhang J, Shi Q, et al. Prevalence, treatment, and associated disability of mental disorders in four provinces in China during 2001-05: an epidemiological survey. Lancet. 2009;373 (9680):2041-2053. doi:10.1016/S0140-6736(09)60660-7

17. Lee S, Leung CM, Kwok KP, Lam K. A community-based study of the relationship between somatic and psychological distress in Hong Kong. Transcult Psychiatry. 2015;52(5):594-615. doi:10.1177/1363461515569756

18. Xiong N, Wei J, Fritzsche K, et al. Psychological and somatic distress in Chinese outpatients at general hospitals: a cross-sectional study. Ann Gen Psychiatry. 2017;16(1):35. doi:10.1186/s12991-017-0158-y

19. Hsu LK, Folstein MF. Somatoform disorders in Caucasian and Chinese Americans. J Nerv Ment Dis. 1997;185(6):382-387. doi:10.1097/00005053-199706000-00004

20. Parker G, Cheah YC, Roy K. Do the Chinese somatize depression? A cross-cultural study. Soc Psychiatry Psychiatr Epidemiol. 2001;36 (6):287-293. doi:10.1007/s001270170046

21. Zung WW, Magruder-Habib K, Velez R, Alling W. The comorbidity of anxiety and depression in general medical patients: a longitudinal study. J Clin Psychiatry. 1990;51:77-80.

22. Hosaka T, Aoki T, Watanabe T, Ishida A. Psychiatric evaluation of rehabilitation patients. Tokai J Exp Clin Med. 1994;19(1-2):7-11.

23. Teymoori A, Real R, Gorbunova A, et al. Measurement invariance of assessments of depression (PHQ-9) and anxiety (GAD-7) across sex, strata and linguistic backgrounds in a European-wide sample of patients after Traumatic Brain Injury. J Affect Disord. 2020;262:278-285. doi:10.1016/j.jad.2019.10.035 
24. Galenkamp H, Stronks K, Snijder MB, Derks EM. Measurement invariance testing of the PHQ-9 in a multi-ethnic population in Europe: the HELIUS study. BMC Psychiatry. 2017;17(1):349. doi:10.1186/s12888-017-1506-9

25. Löwe B, Decker O, Müller S, et al. Validation and standardization of the Generalized Anxiety Disorder Screener (GAD-7) in the general population. Med Care. 2008;46(3):266-274. doi:10.1097/ MLR.0b013e318160d093

26. Cardiovascular Rehabilitation Branch-Chinese Association of Rehabilitation Medicine; Committee of Cardio-Cerebral-Vascular Diseases of Gerontological Society of China. Chinese expert consensus of psychological prescription for patients in cardiovascular department. Zhonghua Xin Xue Guan Bing Za Zhi. 2014;42(1):6-12.

27. Spitzer RL, Kroenke K, Williams JBW, Löwe B. A brief measure for assessing generalized anxiety disorder: the GAD-7. JAMA Intern Med. 2006;166(10):1092-1097. doi:10.1001/archinte.166.10.1092

28. He X, Li C, Qian J, Cui H. Reliability and validity of a generalized anxiety scale in general hospital outpatients. Shanghai Arch Psychiatry. 2010;22(4):200-203.

29. Wang Y, Chen R, Zhang L. Evaluation of the reliability and validity of the generalized anxiety disorder 7-item scale among inpatients in general hospital. J Clin Psychiatry. 2018;28(3):168-171.

30. Zung WWK. A rating instrument for anxiety disorders. Psychosomatics. 1971;12:371-379. doi:10.1016/S0033-3182(71) 71479-0

31. Spitzer RL, Kroenke K, Williams JB. Validation and utility of a self-report version of PRIME-MD: the PHQ primary care study. Primary Care Evaluation of Mental Disorders. Patient Health Questionnaire. JAMA. 1999;282(18):1737-1744. doi:10.1001/ jama.282.18.1737

32. Chen MM, Sheng L, Qu S. Diagnostic test of screening depressive disorders in general hospital with the Patient Health Questionnaire. Chin Ment Henlth J. 2015;29(4):241-245.

33. Zung WWK. A self-rating depression scale. Arch Gen Psychiatry. 1965;12:63-70. doi:10.1001/archpsyc.1965.01720310065008
34. Landis JR, Koch GG. The measurement of observer agreement for categorical data. Biometrics. 1977;33(1):159-174. doi:10.2307/ 2529310

35. Deng BY, Cui JG, Li CJ, et al. Psychological status in 1083 hospitalized patients with coronary artery disease. Zhonghua Xin Xue Guan Bing Za Zhi. 2010;38(8):702-705.

36. Liang JJ, Huang H, Yang B, et al. A cross-sectional survey on the prevalence of anxiety symptoms in Chinese patients with premature ventricular contractions without structural heart disease. Chin Med J. 2012;125(14):2466-2471.

37. Liu MY, Hu DY, Jiang RH, Deng JG, Yang XL. An analysis of psychological problems of patients in cardiology clinic. Zhonghua Nei Ke Za Zhi. 2008;47(4):277-279.

38. Zhang J, Wei J, Shi LL, et al. A multiple center study on depression and anxiety state in the department of neurology. Zhonghua Yi Xue Za Zhi. 2007;87(13):889-893.

39. Zheng LJ, Ma JC, Fang D, et al. The quantification and assessment of depression and anxiety in patients with postoperative gastroparesis syndrome. Ther Clin Risk Manag. 2018;14:551-556. doi:10.2147/ TCRM.S155358

40. Kroenke K, Spitzer RL, Williams Janet BW. The Patient Health Questionnaire-2: validity of a Two-Item Depression Screener. Med Care. 2003;41(11):1284-1292. doi:10.1097/01. MLR.0000093487.78664.3C

41. Zhang YN, Jin C, Ning L, Gong S, Xiao Y, Yu GL. Clinical characteristics and related factors of somatization symptoms in outpatients with psychiatric disorders of the department of cardiology in general hospitals. Zhonghua Xin Xue Guan Bing Za Zhi. 2019;47 (11):907-912. doi:10.3760/cma.j.issn.0253-3758.2019.11.010

42. Becker PM. Insomnia: prevalence, impact, pathogenesis, differential diagnosis, and evaluation. Psychiatr Clin North Am. 2006;29 (4):855-870. doi:10.1016/j.psc.2006.08.001
Therapeutics and Clinical Risk Management

\section{Publish your work in this journal}

Therapeutics and Clinical Risk Management is an international, peerreviewed journal of clinical therapeutics and risk management, focusing on concise rapid reporting of clinical studies in all therapeutic areas, outcomes, safety, and programs for the effective, safe, and sustained use of medicines. This journal is indexed on PubMed Central, CAS,
EMBase, Scopus and the Elsevier Bibliographic databases. The manuscript management system is completely online and includes a very quick and fair peer-review system, which is all easy to use. Visit http://www.dovepress.com/testimonials.php to read real quotes from published authors. 